\title{
Atendimentos de emergência por acidentes na Rede de Vigilância de Violências e Acidentes - Brasil, 2006
}

\author{
Unintentional injuries \\ at theEmergency Department Injury SurveillanceSystem - Brazil, 2006
}

\author{
M árcio D ênis $M$ edeiros $M$ ascarenhas ${ }^{1}$ \\ M arta M aria Alves da Silva ${ }^{1}$ \\ Deborah Carvalho M alta ${ }^{1}$ \\ Lenildo de M oura ${ }^{1}$ \\ Vilma Pinheiro Gawryszewski ${ }^{2}$ \\ Valter Chaves Costa ${ }^{1}$ \\ $\mathrm{M}$ aria de Fátima $\mathrm{M}$ arinho Souza ${ }^{3}$ \\ Otaliba Libânio de M orais N eto ${ }^{4}$
}

${ }^{1}$ Coordenação Geral de

Vigilância deDoençase

Agravos Não

Transmissíveis, Secretaria deVigilância em Saúde,

Ministério da Saúde. SAF

Sul, Trecho 02, Lotes 05 e

06, Bloco F, Torre I, Edifício

Premium, Térreo, Sala 14.

70070-600 Brasília DF.

marcio.mascarenhas@

saude.gov.br

${ }^{2}$ Secretaria deEstado da

SaúdedeSão Paulo.

${ }^{3}$ Coordenação Geral de

Informação eAnálise.

${ }^{4}$ Departamento deAnálise deSituação deSaúde.
Abstract In 2006, the Brazilian M inistry of $\mathrm{H}$ ealth established the I njury Surveillance System N etwork in Sentinel Services (RedeVIVA) to describe data on injuries victims treated at emergency departments (ED), especially minor injuries which do not cause deaths and hospitalization. This study describes the characteristics of unintentional injuries victims treated in these EDs by means of a transversal descriptivestudy with data coming from ED s located in 35 municipalities with the highest injury rates. The data were collected during 30 consecutive days in an alternated 12-hour shift. There werea total of 41,677 patients with unintentional injuries, mainly among males (64.8\%), people aged 20 to 29 years $(23.0 \%)$ and low level of education (45.8\%). Falls were the main cause ( $40.1 \%$ overall), followed by transport related injuries ( $26.6 \%$ overall). The more frequent place of occurrence were public streets (36.3\% overall) and residences ( $33.6 \%$ overall). The body parts most affected were upper members $(42.7 \%)$, lower members (42.0\%) and head/face (29.2\%). Theinjury surveillance in sentinel services allows collecting timeless data about minor cases which is essential for planning and implementing preventive measures. Key words Accidents, Violence, Injuries, External causes, Sentinel surveillance
Resumo Em 2006, o M inistério da Saúde implantou a Rede de Serviços Sentinelas de Vigilância de Violências eAcidentes (RedeVIVA) a fim de descre ver os atendimentos de emergência por violências e acidentes, principalmente as lesões de menor gravidadequenão implicam mortes ou internações. $N$ este artigo, descrevem-se as características dos atendimentos de emergência por acidentes realizados nesses serviços. Estudo descritivo, de corte transversal, realizado em 35 municípios com os maiores coeficientes de morbimortalidade por causas externas. Os dados foram coletados durantetrinta dias consecutivos em plantões alternados de doze horas. Registraram-se 41.677 atendimentos por acidentes, com pre domínio dehomens $(64,8 \%$ ), pessoas de 20 a 29 anos de idade (22,9\%) e com baixa escolaridade (56,8\%). Asquedas (40,2\%) foram a principal causa, seguidas dos acidentes de transporte (26,6\%). Os locais de ocorrência maisfrequentesforam via pública (36,2\%) e residência (33,7\%). As partes do corpo mais atingidas foram membros superiores (38,1\%), membros inferiores (37,6\%) e cabeça/face (25,7\%). A vigilância sentinela de violências e acidentes permite coletar dados em tempo hábil sobre eventos menos graves, cujo conhecimento éfundamental para o planejamento de medidas preventivas.

Palavras-chave Acidentes, Violência, Lesões, Causas externas, Vigilância de evento sentinela 
Introdução

As causas externas podem ser classificadas como lesões intencionais (violências), representadas pelas agressões, homicídios, suicídios, privação ou negligência, elesões não intencionais, queincluem os acidentes de transporte, afogamentos, quedas, queimaduras, dentre outros ${ }^{1}$. Elas são responsáveis por grande parte dos óbitos em todo o mundo, especialmente nos países em desenvolvimento ${ }^{2,3}$ como o Brasil, onde as causas externas ocupam a terceira posição ${ }^{4}$.

No âmbito da saúde pública, a prevenção destes agravos tem como etapa inicial a descrição de sua magnitude e impacto ${ }^{2,3}$. No Brasil, essa descrição é realizada a partir do Sistema de Informações sobre M ortalidade (SIM) e do Sistema de Informações H ospitalares (SIH), nos quais são registrados os principais tipos de causas externas que determinam mortes e internações, respectivamente. Em 2005 , foram registradas 127.633 mortes por causas externas ( $12 \%$ do total de mortes no país), com coeficiente de mortalidade de 69 por 100 mil habitantes. No mesmo ano, 11,4 milhões deinternações foram realizadas nos hospitais credenciados ao Sistema Ú nico de Saúde (SU S). D estas, 788.701 (7\%) foram decorrentes das causas externas, com custo estimado em R $\$ 594$ milhões, o que representou $8,5 \%$ do total pago para todas as internações ${ }^{4}$.

Embora os dados disponíveis no $\mathrm{SIM}$ e $\mathrm{SIH}$ sejam úteis para monitorar a vigilância epidemiológica das causas externas, esses sistemas não fornecem dados sobre as lesões de menor gravidade, que não implicam mortes ou internações, mas que são responsáveis por uma grande demanda de atendimentos nos serviços de emergência. Para preencher essa lacuna, o M inistério da Saúde implantou, em 2006, a Redede Serviços Sentinelas de Vigilância de Violências e Acidentes (RedeVIVA), estruturada em dois componentes: 1) vigilância de violências e acidentes em emergências hospitalares, que visa à caracterização dos atendimentos de emergência por causas externas; e2) vigilância das violências sexual, doméstica e/ ou outras violências interpessoais, em cumprimento às exigências legais denotificação da violência contra criança, adolescente, mulher eidoso. Esta estratégia de vigilância pretende conhecer a distribuição, magnitude, tendência efatores de risco das violências eacidentes, além deidentificar condicionantes sociais, econômicos e ambientais de sua ocorrência para subsi diar o planejamento ea aplicação deinterven ções apropriadas de prevenção e controle 5 .

0 presente estudo tem como objetivo descre ver as características dos atendimentos de emer- gência por acidentes registrados em serviços integrantes da Rede VIVA em 2006.

\section{Métodos}

Estudo descritivo, de corte transversal, realizado em 65 serviços de emergência credenciadosao SUS, localizados em 34 municípios e Distrito Federal, os quais atenderam aos seguintes critérios de inclusão: elevado coeficiente de morbimortalidade por causas externas, existência deserviços de referência para 0 atendimento às vítimas de violências e acidentes, execução de ações intersetoriais de prevenção das violências e acidentes 5 .

Considerando a metodologia de amostragem por conveniência, a população de estudo foi composta pelas vítimas deviolências eacidentes que procuraram atendimento nos serviços de emergência dosmunicípios selecionados durantetrinta diasconsecutivos entreos meses de setembro enovembro de 2006. A escolha deum único mês deveu-seà tentativa deevitar a interferência dos mesestípicos deférias e festas de fim de ano sobre a sazonalidade e maior ocorrência das causas externas. Devido ao grande número de atendimentos nos serviços de emergência, decidiu-se pela coleta de dados em plantões de doze horas e em turnos alternados, o que facilitou 0 trabalho de campo e permitiu estabelecer a representatividade dos atendimentos em cada serviço.

Utilizou-se formulário padronizado que continha os seguintes grupos de variáveis: identificação do paciente (nome, idade, sexo, raça/cor da pele, escolaridade, meio detransporteutilizado para chegar ao hospital), tipo de ocorrência (acidente ou violência), local deocorrência (residência, escola, via pública, trabalho, bar, local de prática de esporte), tipo de acidente (acidente de transporte, queda, queimadura, outros), tipo de violência (agressão, maus-tratos, tentativa de suicídio), características do agressor (sexo, relação com a víti$\mathrm{ma}$ ), parte do corpo atingida e evolução dos casos (alta, hospitalização, óbito).

Os dados foram coletados por técnicos das secretarias de saúde dos municípiosincluídosna pesquisa e em parceria com representantes das respectivas secretarias estaduais de saúde. Todos os participantes da coleta de dados receberam treinamento específico sobre vigilância de violências e acidentes, durante 0 qual foram apresentados os seguintes aspectos: operacionalização do sistema de vigilância, seleção dos serviços de emergência, padronização de definições, uniformização das técnicas de obtenção e registro de dados, fluxo de envio dos dados (Figura 1). 


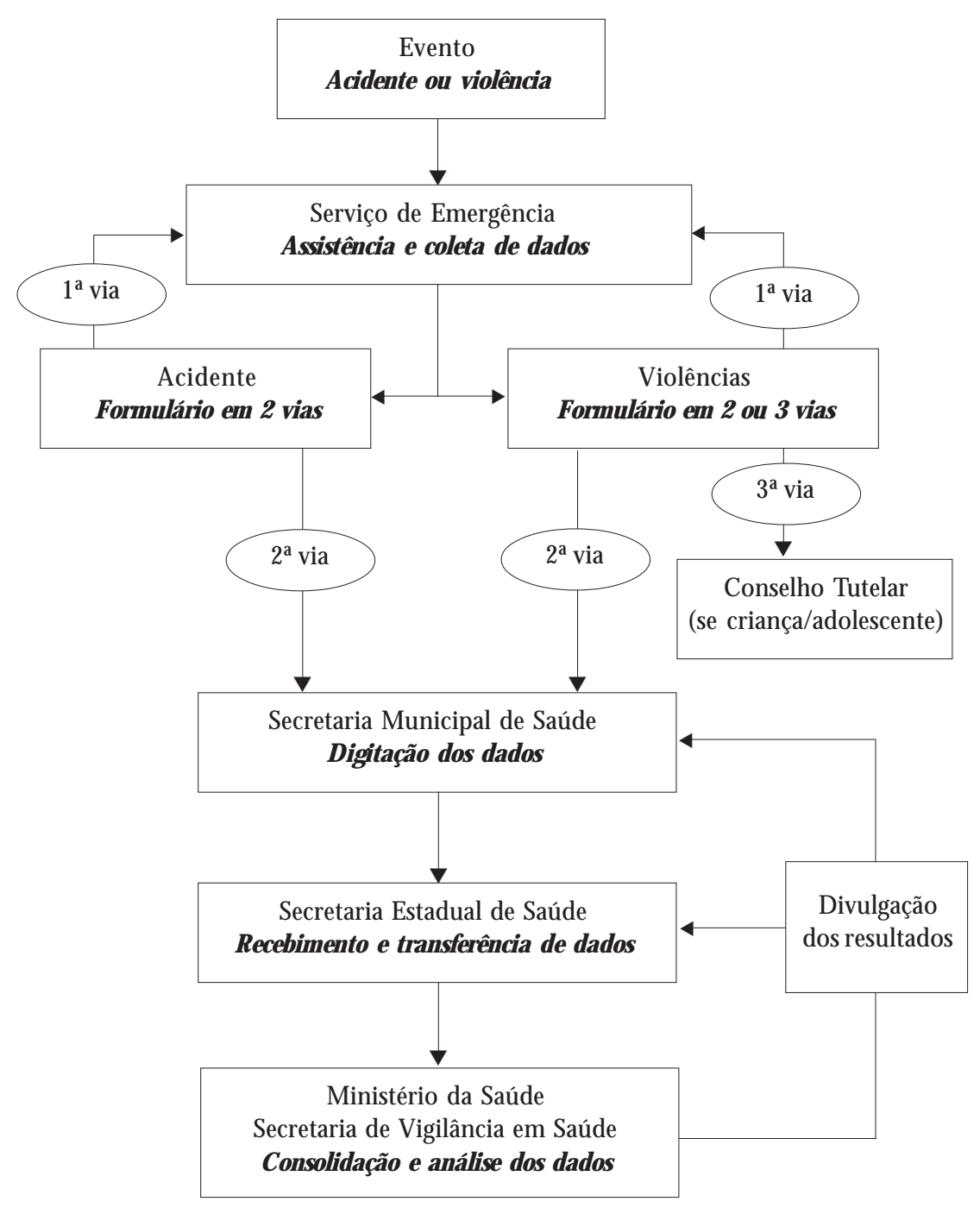

Figura 1. Fluxograma de coleta, processamento e divulgação dos dados da vigilância de violências e acidentes em serviços sentinelas. M unicípios selecionados - Brasil, 2006.

Os atendimentos foram classificados em dois grupos: acidentes eviolências. Acidente foi definido como "evento não intencional e evitável, causador de lesões físicas e emocionais, no âmbito doméstico ou social como trabalho, escola, esportee lazer" 6 . I ncluíram-se neste grupo os acidentes de transporte, quedas, queimaduras, cortes, queda de objetos sobre a pessoa, envenenamento, sufocação, afogamento, dentre outros. Considerou-se como violência "o uso da força contra um grupo ou uma comunidade, que resulte ou tenha qualquer possibilidadederesultar em lesão, morte, dano psicológico, deficiência de desenvolvimento ou privação" ${ }^{3}$. Sob esta denominação, incluíram-se as tentativas de suicídio, maus-tratos e agressões.

Os dados foram digitados no Setor de Vigilância Epidemiológica de cada município participante da pesquisa e, posteriormente, transferidos para o M inistério da Saúde, onde se procedeu a consolidação da base de dados nacional, análise e divulgação dos resultados. U tilizaram-se os programas Link Plus e Epi Info 3.3.27 para a identificação de registros duplicados e análise de dados, respectivamente. 0 banco de dados nacional continha 
47.554 registros, dos quais foram identificados e excluídos 759 (1,6\%) por estarem duplicados. Os resultados aqui apresentados referem-se apenas aos atendimentos de emergência por acidentes.

$\mathrm{O}$ projeto de pesquisa foi aprovado pelo Comitê Nacional de Ética em Pesquisa. Por se tratar de ação específica de vigilância epidemiológica de âmbito nacional, a assinatura do termo de consentimento livre e esclarecido foi substituída por consentimento verbal, obtido pelo paciente ou por seu responsável. Foi garantido total anonimato e privacidade aos pacientes, profissionais e gestores dos serviços onde a pesquisa foi realizada, assim como a liberdade para desistir de participar da entrevista a qualquer momento, sem prejuízo de qualquer natureza para si próprio ou familiares.

\section{Resultados}

Foram registrados 46.795 atendimentos, dos quais $41.677(89,1 \%)$ foram classificados como acidentes. Destes, $26.989(64,8 \%)$ ocorreram no sexo masculino e 14.688 ( $35,2 \%)$, no sexo feminino. A Tabela 1 apresenta a distribuição dos atendimentos de emergência por acidentes em todos os municípios que realizaram a pesquisa em 2006.

As maiores proporções de atendimentos foram observadas em pessoas jovens, apresentando distribuição diferente entreossexos. Entreos homens, a faixa etária mais frequenteincluiu pessoas de 20 a 29 anos (25,4\%), seguidas das de 10 a 19 anos $(19,4 \%)$ e de 0 a 9 anos (18,8\%). Nas mulheres, a maior proporção de atendimentos compreendeu as pessoas de 0 a 9 anos (22\%), seguidas das de 10 a 19 anos (18,9\%) e de 20 a 29 anos (18,2\%). Com relação à raça/cor da pele, os pardos representaram $49,2 \%$, seguidos de brancos ( $35,9 \%$ ) e pretos $(11,2 \%)$, enquanto amarelos (1,3\%) e indígenas $(0,2 \%)$ corresponderam às menores proporções no total de atendimentos. Esta distribuição foi seme Ihante quando desagregada por sexo da vítima. Observou-se que $56,8 \%$ dos pacientes informaram ter cursado de um a oito anos de estudo (ensino fundamental) e $17,9 \%$ cursaram de nove a onze anos de estudo (ensino médio), sendo as menores proporções constituídas por pessoas sem escolaridade $(4,3 \%)$ e os que concluíram o ensino superior $(1,8 \%)$. Os meios detran sporte maisutilizados para chegar ao serviço deemergênciaforam veículo particular (38\%) e ambulância (27,1\%), incluindo as unidades do Serviço deAtendimento Móvel deUrgência (SAM U), utilizadas em $14 \%$ dos atendimentos (Tabela 2).
Segundo o tipo de acidente, destacaram-se as quedas ( $n=16.768 ; 40,2 \%$ ), acidentes de transporte ( $n=11.080 ; 26,6 \%$ ) e queimaduras ( $n=976 ; 2,3 \%$ ). As quedas do mesmo nível foram responsáveis por mais da metade $(59,5 \%)$ deste tipo de acidente em ambos os sexos. A maior parte dos acidentes de transporte envolveu motocicleta $(46,2 \%)$, bicicleta $(22,5 \%)$ e automóvel $(22 \%)$, sendo o condutor 0 tipo de vítima mais frequente (58,2\%). Entre os homens, a principal vítima também foi o condutor $(68,4 \%)$; entre as mulheres, destacaram-se as passageiras (44,3\%). Dos acidentes por queimaduras, substância quente (51,3\%) foi o agente responsável pela maioria dos atendimentos nesse grupo, principalmenteentreas mulheres (63,1\%). O utrosagentes, como substâncias químicas (ácidos e bases) e superfícies quentes (escapamento de moto, utensílios de cozinha), causaram $19 \%$ das queimaduras. Os demais tipos de acidentes foram responsáveis por cerca de um terço do total de atendimentos $(n=12.853 ; 30,8 \%)$. Dentre eles, destacaram-se os acidentes envolvendo material pérfuro-cortante (20,3\%) equeda de objetos sobre a pessoa ( $11,8 \%$ ). Ainda foram identificados, nesta categoria, os acidentes por arma de fogo, afogamento e sufocação. Outros tipos de acidentes incluíram ataques de animais (picadas, mordeduras), introdução/aspiração de corpo estranho, colisões entre pessoas ou entre pessoas e objetos inanimados, além de entorses/ luxação, perfazendo, em seu conjunto, $24 \%$ dos atendimentos do grupo. $\mathrm{Em} 39,9 \%$ dos registros, 0 evento foi um acidente, masnão teve seu tipo especificado (Tabela 3).

Quanto ao horário de ocorrência dos acidentes, observou-se maior frequência no período diurno (manhã etarde), principalmenteà tarde (37,1\%). Segundo o local de ocorrência, 36,2\% dosacidentes aconteceram em via pública, incluindo ruas erodovias, e proporção semelhante aconteceu em residências (33,7\%). Entre os homens, os acidentes ocorreram em maior proporção na via pública (38,8\%), enquanto o domicílio ( $46,7 \%)$ foi o local mais frequente para a ocorrência dos acidentes entre as mulheres. Referência ao uso deálcool ou drogasilícitas foi observada em $8,1 \%$ detodos os eventos e em $10,6 \%$ dos acidentes registrados entre os homens. As regiões do corpo mais frequentemente atingidas foram os membros superiores $(38,1 \%)$, membros inferiores $(37,6 \%)$ e cabeça/face $(25,7 \%)$. Quanto à evolução dos casos após o atendimento deemergência inicial, 64,4\% receberam alta, 17,9\% foram encaminhados para internação hospitalar e $13,6 \%$ foram direcionados ao acompanhamento ambulatorial. 0 percentual geral de óbitos nesses serviços foi de 0,2\% (Tabela 4). 
Tabela 1. Distribuição dos atendimentos de emergência por acidentes segundo municípios selecionados para a implantação da vigilância de acidentes em serviços sentinelas. M unicípios selecionados - Brasil, 2006.

\begin{tabular}{|c|c|c|c|c|c|c|}
\hline \multirow{2}{*}{ Município* } & \multicolumn{2}{|c|}{ Total } & \multicolumn{2}{|c|}{ M asculino } & \multicolumn{2}{|c|}{ Feminino } \\
\hline & $\mathrm{n}$ & $\%$ & $\mathrm{n}$ & $\%$ & $\mathrm{n}$ & $\%$ \\
\hline Alta Floresta - M T & 195 & 0,5 & 144 & 0,5 & 51 & 0,3 \\
\hline Aracaju - SE & 1.906 & 4,6 & 1.288 & 4,8 & 618 & 4,2 \\
\hline Belo Horizonte - MG & 2.318 & 5,6 & 1.481 & 5,5 & 837 & 5,7 \\
\hline Brasília - DF & 1.336 & 3,2 & 863 & 3,2 & 473 & 3,2 \\
\hline Campinas - SP & 596 & 1,4 & 402 & 1,5 & 194 & 1,3 \\
\hline Campo Grande - MS & 751 & 1,8 & 470 & 1,7 & 281 & 1,9 \\
\hline Cuiabá - MT & 1.547 & 3,7 & 992 & 3,7 & 555 & 3,8 \\
\hline Curitiba - PR & 1.952 & 4,7 & 1.192 & 4,4 & 760 & 5,2 \\
\hline Diadema - SP & 1.211 & 2,9 & 770 & 2,9 & 441 & 3,0 \\
\hline Florianópolis - SC & 1.459 & 3,5 & 867 & 3,2 & 592 & 4,0 \\
\hline Fortaleza - CE & 1.017 & 2,4 & 657 & 2,4 & 360 & 2,5 \\
\hline Goiânia - GO & 2.204 & 5,3 & 1.502 & 5,6 & 702 & 4,8 \\
\hline Guarulhos - SP & 1.193 & 2,9 & 703 & 2,6 & 490 & 3,3 \\
\hline João Pessoa - PB & 1.866 & 4,5 & 1.196 & 4,4 & 670 & 4,6 \\
\hline Jundiaí - SP & 862 & 2,1 & 573 & 2,1 & 289 & 2,0 \\
\hline M aceió - AL & 1.761 & 4,2 & 1.157 & 4,3 & 604 & 4,1 \\
\hline M anaus - AM & 306 & 0,7 & 201 & 0,7 & 105 & 0,7 \\
\hline Natal - RN & 872 & 2,1 & 611 & 2,3 & 261 & 1,8 \\
\hline Olinda - PE & 424 & 1,0 & 266 & 1,0 & 158 & 1,1 \\
\hline Palmas - TO & 378 & 0,9 & 250 & 0,9 & 128 & 0,9 \\
\hline Porto Velho - RO & 519 & 1,2 & 355 & 1,3 & 164 & 1,1 \\
\hline Recife - PE & 3.214 & 7,7 & 1.934 & 7,2 & 1.280 & 8,7 \\
\hline Ribeirão Preto - SP & 343 & 0,8 & 243 & 0,9 & 100 & 0,7 \\
\hline Rio Branco - AC & 1.138 & 2,7 & 734 & 2,7 & 404 & 2,8 \\
\hline Rio de Janeiro - RJ & 727 & 1,7 & 480 & 1,8 & 247 & 1,7 \\
\hline Salvador - BA & 1.505 & 3,6 & 996 & 3,7 & 509 & 3,5 \\
\hline Santo André - SP & 842 & 2,0 & 567 & 2,1 & 275 & 1,9 \\
\hline São José do Rio Preto - SP & 1.234 & 3,0 & 866 & 3,2 & 368 & 2,5 \\
\hline São Luís - MA & 3.426 & 8,2 & 2.114 & 7,8 & 1.312 & 8,9 \\
\hline Serra - ES & 719 & 1,7 & 517 & 1,9 & 202 & 1,4 \\
\hline Sobral - CE & 801 & 1,9 & 526 & 1,9 & 275 & 1,9 \\
\hline Sorocaba - SP & 1.370 & 3,3 & 899 & 3,3 & 471 & 3,2 \\
\hline Teresina - PI & 727 & 1,7 & 522 & 1,9 & 205 & 1,4 \\
\hline Várzea Grande - M T & 740 & 1,8 & 513 & 1,9 & 227 & 1,5 \\
\hline Vitória - ES & 218 & 0,5 & 138 & 0,5 & 80 & 0,5 \\
\hline Total & 41.677 & 100,0 & 26.989 & 100,0 & 14.688 & 100,0 \\
\hline
\end{tabular}

*Não foram apresentados os dados de quatro municípios queatendiam aos critérios deinclusão, mas não realizaram a pesquisa em 2006.

\section{Discussão}

A vigilância epidemiológica de causas externas é uma ação relevante para a sociedade, pois, além de permitir o monitoramento e a análise de possíveis mudanças no perfil desses agravos, contribui para a educação e o planejamento de ações intersetoriais de prevenção de violências e acidentes².
O propósito do estabelecimento da vigilância de acidentes em serviços deemergênciaé possibilitar 0 acesso às informações acerca das causas externas ainda pouco conhecidas, de alta ocorrência e impacto na saúde da população. Os resultados ora apresentados são fundamentais para auxiliar no real dimensionamento dos acidentes ocorridos em alguns municípios brasileiros, muitas vezesina- 
Tabela 2. Distribuição dos atendimentos de emergência por acidentes segundo características das vítimas. M unicípios selecionados - Brasil, 2006.

\begin{tabular}{|c|c|c|c|c|c|c|}
\hline \multirow{2}{*}{ Características } & \multicolumn{2}{|c|}{ Total } & \multicolumn{2}{|c|}{ Masculino } & \multicolumn{2}{|c|}{ Feminino } \\
\hline & $n$ & $\%$ & $n$ & $\%$ & $\mathrm{n}$ & $\%$ \\
\hline \multicolumn{7}{|l|}{ Faixa etária (anos) } \\
\hline $0-9$ & 8.309 & 19,9 & 5.074 & 18,8 & 3.235 & 22,0 \\
\hline $10-19$ & 8.000 & 19,2 & 5.230 & 19,4 & 2.770 & 18,9 \\
\hline $20-29$ & 9.544 & 22,9 & 6.868 & 25,4 & 2.676 & 18,2 \\
\hline $30-39$ & 6.216 & 14,9 & 4.343 & 16,1 & 1.873 & 12,8 \\
\hline $40-49$ & 4.249 & 10,2 & 2.750 & 10,2 & 1.499 & 10,2 \\
\hline $50-59$ & 2.470 & 5,9 & 1.390 & 5,2 & 1.080 & 7,4 \\
\hline$\geq 60$ & 2.757 & 6,6 & 1.240 & 4,6 & 1.517 & 10,3 \\
\hline $\bar{N}$ ão informado & 132 & 0,3 & 94 & 0,3 & 38 & 0,3 \\
\hline \multicolumn{7}{|l|}{ Raça/cor } \\
\hline Parda & 20.493 & 49,2 & 13.604 & 50,4 & 6.889 & 46,9 \\
\hline Branca & 14.979 & 35,9 & 9.150 & 33,9 & 5.829 & 39,7 \\
\hline Preta & 4.661 & 11,2 & 3.210 & 11,9 & 1.451 & 9,9 \\
\hline Amarela & 528 & 1,3 & 342 & 1,3 & 186 & 1,3 \\
\hline Indígena & 82 & 0,2 & 62 & 0,2 & 20 & 0,1 \\
\hline Não informado & 934 & 2,2 & 621 & 2,3 & 313 & 2,1 \\
\hline \multicolumn{7}{|l|}{ Escolaridade } \\
\hline Sem escolaridade & 1.783 & 4,3 & 1.015 & 3,8 & 768 & 5,2 \\
\hline 1 - 8 (ensino fundamental) & 23.666 & 56,8 & 15.727 & 58,3 & 7.939 & 54,1 \\
\hline 9 - 11 (ensino médio) & 7.467 & 17,9 & 4.774 & 17,7 & 2.693 & 18,3 \\
\hline 12 e mais (ensino superior) & 762 & 1,8 & 418 & 1,5 & 344 & 2,3 \\
\hline Não se aplica & 4.861 & 11,7 & 2.892 & 10,7 & 1.969 & 13,4 \\
\hline Não informado & 3.138 & 7,5 & 2.163 & 8,0 & 975 & 6,6 \\
\hline \multicolumn{7}{|c|}{ M eio de transporte para chegar ao hospital } \\
\hline Veículo particular & 15.819 & 38,0 & 9.946 & 36,9 & 5.873 & 40,0 \\
\hline Ambulância* & 11.294 & 27,1 & 7.997 & 29,7 & 3.297 & 22,4 \\
\hline Coletivo & 7.436 & 17,8 & 4.327 & 16,0 & 3.109 & 21,2 \\
\hline A pé & 2.157 & 5,2 & 1.336 & 5,0 & 821 & 5,6 \\
\hline Viatura da polícia & 398 & 1,0 & 293 & 1,1 & 105 & 0,7 \\
\hline Outro & 3.458 & 8,3 & 2.371 & 8,8 & 1.087 & 7,4 \\
\hline Não informado & 1.115 & 2,7 & 719 & 2,7 & 396 & 2,7 \\
\hline Total & 41.677 & 100,0 & 26.989 & 100,0 & 14.688 & 100,0 \\
\hline
\end{tabular}

*Inclui unidades de atendimento pré-hospitalar do Serviço deAtendimento M óvel deU rgência (SAMU) eambulâncias deoutros serviços, como clínicas, hospitais ou seguradoras de saúde.

cessíveis pelos gestores e tomadores de decisão, quer seja por falhas no atual modelo de vigilância, quer seja pela limitação dos sistemas de informação atualmente disponíveis, cujo foco são os acidentes mais graves e que resultam em internações ou óbitos captados pelo SIH ou SIM, respectivamente. Outra fonte de dados para monitorar os atendimentos por acidentes é o Sistema de Informações Ambulatoriais (SIA); porém, este sistema não apresenta dados suficientes à análise epidemiológica, sendo mais útil ao acompanhamento financeiro dos procedimentos pagos pelo SUS no atendimento ambulatorial ${ }^{4,8}$. Além disso, a difi- culdade em monitorar os atendimentos de emergência por acidentes decorre da própria natureza do evento, que exige rápido diagnóstico eintervenção oportunos por parte da equipe de assistência.

No presente estudo, o predomínio de homens entre as vítimas (razão entre sexos masculino e feminino igual a 1,8 ) não pode ser justificado por um fator biológico específico, mas, talvez, pelos padrões socioculturais cristalizados na noção de gêner ${ }^{9}$. Somenteem 2005, a população masculina representou $83,6 \%$ de toda a mortalidade por causas externas, com coeficiente de 117,6 mortes para cada grupo de 100 mil homens, cinco vezes maior 
Tabela 3. Distribuição dos atendimentos de emergência por acidentes segundo tipo de acidente. M unicípios selecionados - Brasil, 2006.

\begin{tabular}{|c|c|c|c|c|c|c|}
\hline \multirow{2}{*}{ Tipo de acidente } & \multicolumn{2}{|c|}{ Total } & \multicolumn{2}{|c|}{ Masculino } & \multicolumn{2}{|c|}{ Feminino } \\
\hline & $\mathrm{n}$ & $\%$ & $\mathrm{n}$ & $\%$ & $\mathrm{n}$ & $\%$ \\
\hline \multicolumn{7}{|l|}{ Quedas } \\
\hline Mesmo nível & 9.976 & 59,5 & 5.384 & 56,7 & 4.592 & 63,1 \\
\hline Escada & 1.788 & 10,7 & 893 & 9,4 & 895 & 12,3 \\
\hline Leito/sofá/berço & 900 & 5,4 & 451 & 4,8 & 449 & 6,2 \\
\hline Outras alturas & 3.409 & 20,3 & 2.336 & 24,6 & 1.073 & 14,7 \\
\hline Não informado & 695 & 4,1 & 428 & 4,5 & 267 & 3,7 \\
\hline Subtotal & 16.768 & 100,0 & 9.492 & 100,0 & 7.276 & 100,0 \\
\hline \multicolumn{7}{|l|}{ Acidentes de transporte } \\
\hline \multicolumn{7}{|l|}{ M eio de transporte } \\
\hline M otocicleta & 5.116 & 46,2 & 4.040 & 49,6 & 1.076 & 36,7 \\
\hline Bicicleta & 2.496 & 22,5 & 1.857 & 22,8 & 639 & 21,8 \\
\hline Automóvel & 2.433 & 22,0 & 1.594 & 19,6 & 839 & 28,6 \\
\hline Coletivo & 345 & 3,1 & 158 & 1,9 & 187 & 6,4 \\
\hline Outros & 317 & 2,9 & 255 & 3,1 & 62 & 2,1 \\
\hline $\mathrm{N}$ ão informado & 373 & 3,4 & 241 & 3,0 & 132 & 4,5 \\
\hline Subtotal & 11.080 & 100,0 & 8.145 & 100,0 & 2.935 & 100,0 \\
\hline \multicolumn{7}{|l|}{ Tipo de vítima } \\
\hline Condutor & 6.446 & 58,2 & 5.575 & 68,4 & 871 & 29,7 \\
\hline Passageiro & 2.477 & 22,4 & 1.177 & 14,5 & 1.300 & 44,3 \\
\hline Pedestre & 1.788 & 16,1 & 1.133 & 13,9 & 655 & 22,3 \\
\hline Outro & 51 & 0,5 & 46 & 0,6 & 5 & 0,2 \\
\hline Não informado & 318 & 2,9 & 214 & 2,6 & 104 & 3,5 \\
\hline Subtotal & 11.080 & 100,0 & 8.145 & 100,0 & 2.935 & 100,0 \\
\hline \multicolumn{7}{|l|}{ Queimaduras } \\
\hline Substância quente & 501 & 51,3 & 229 & 42,0 & 272 & 63,1 \\
\hline Fogo & 179 & 18,3 & 111 & 20,4 & 68 & 15,8 \\
\hline Choque elétrico & 57 & 5,8 & 40 & 7,3 & 17 & 3,9 \\
\hline Outros & 184 & 18,9 & 123 & 22,6 & 61 & 14,2 \\
\hline Não informado & 55 & 5,6 & 42 & 7,7 & 13 & 3,0 \\
\hline Subtotal & 976 & 100,0 & 545 & 100,0 & 431 & 100,0 \\
\hline \multicolumn{7}{|l|}{ Outros acidentes } \\
\hline Corte & 2.611 & 20,3 & 1931 & 21,9 & 680 & 16,8 \\
\hline Queda de objetos sobre a pessoa & 1.524 & 11,9 & 1.107 & 12,6 & 417 & 10,3 \\
\hline Envenenamento & 377 & 2,9 & 214 & 2,4 & 163 & 4,0 \\
\hline Arma de fogo & 80 & 0,6 & 65 & 0,7 & 15 & 0,4 \\
\hline Afogamento & 24 & 0,2 & 18 & 0,2 & 6 & 0,1 \\
\hline Sufocação & 22 & 0,2 & 13 & 0,1 & 9 & 0,2 \\
\hline Outros & 3.088 & 24,0 & 1.977 & 22,4 & 1.111 & 27,5 \\
\hline Não especificado & 5.127 & 39,9 & 3.482 & 39,5 & 1.645 & 40,7 \\
\hline Subtotal & 12.853 & 100,0 & 8.807 & 100,0 & 4.046 & 100,0 \\
\hline Total & 41.677 & 100,0 & 26.989 & 100,0 & 14.688 & 100,0 \\
\hline
\end{tabular}

do que entre a população feminina (22,4/100 mil). Distribuição semelhante foi observada com relação à morbidade: de todas as internações por causas externas ocorridas em 2005, 70,3\% eram de pacientes do sexo masculino ${ }^{4}$.
Adolescentes eadultosjovenstêm sido, frequentemente, identificados como as principais vítimas de causas externas $5^{9,10}$. Fatores como inexperiência, busca de emoções, prazer em experimentar situações de risco, impulsividade e abuso de substâncias 
Tabela 4. Distribuição dos atendimentos de emergência por acidentes segundo características do evento. M unicípios selecionados - Brasil, 2006.

\begin{tabular}{|c|c|c|c|c|c|c|}
\hline \multirow{2}{*}{ Características } & \multicolumn{2}{|c|}{ Total } & \multicolumn{2}{|c|}{ Masculino } & \multicolumn{2}{|c|}{ Feminino } \\
\hline & $\mathrm{n}$ & $\%$ & $n$ & $\%$ & $\mathrm{n}$ & $\%$ \\
\hline \multicolumn{7}{|l|}{ H ora da ocorrência (turno) } \\
\hline 06:00 - 11:59 (manhã) & 10.551 & 25,3 & 6.630 & 24,6 & 3.921 & 26,7 \\
\hline $12: 00$ - 17:59 (tarde) & 15.475 & 37,1 & 10.160 & 37,6 & 5.315 & 36,2 \\
\hline 18:00 - 23:59 (noite) & 11.132 & 26,7 & 7.215 & 26,7 & 3.917 & 26,7 \\
\hline 00:00 - 05:59 (madrugada) & 2.985 & 7,2 & 1.993 & 7,4 & 992 & 6,8 \\
\hline Não informado & 1.534 & 3,7 & 991 & 3,7 & 543 & 3,7 \\
\hline \multicolumn{7}{|l|}{ Local de ocorrência } \\
\hline Via pública (ruas/rodovias) & 15.104 & 36,2 & 10.465 & 38,8 & 4.639 & 31,6 \\
\hline Residência & 14.044 & 33,7 & 7.190 & 26,6 & 6.854 & 46,7 \\
\hline Trabalho & 4.931 & 11,8 & 3.969 & 14,7 & 962 & 6,5 \\
\hline Escola & 1.975 & 4,7 & 1.119 & 4,1 & 856 & 5,8 \\
\hline Local de esporte & 1.960 & 4,7 & 1.756 & 6,5 & 204 & 1,4 \\
\hline Bar ou similar & 310 & 0,7 & 225 & 0,8 & 85 & 0,6 \\
\hline Outros & 2.369 & 5,7 & 1.608 & 6,0 & 761 & 5,2 \\
\hline Não informado & 984 & 2,4 & 657 & 2,4 & 327 & 2,2 \\
\hline \multicolumn{7}{|l|}{ U so de álcool/drogas } \\
\hline Sim & 3.377 & 8,1 & 2.858 & 10,6 & 519 & 3,5 \\
\hline Não & 36.055 & 86,5 & 22.582 & 83,7 & 13.473 & 91,7 \\
\hline Não informado & 2.245 & 5,4 & 1.549 & 5,7 & 696 & 4,7 \\
\hline \multicolumn{7}{|l|}{ Local da lesão* } \\
\hline M embros superiores & 15.880 & 38,1 & 10.634 & 39,4 & 5.246 & 35,7 \\
\hline Membros inferiores & 15.668 & 37,6 & 9.860 & 36,5 & 5.808 & 39,5 \\
\hline Cabeça/face & 10.720 & 25,7 & 7.455 & 27,6 & 3.265 & 22,2 \\
\hline Tórax/dorso & 2.821 & 6,8 & 1.953 & 7,2 & 868 & 5,9 \\
\hline Abdome/quadril & 1.735 & 4,2 & 1.064 & 3,9 & 671 & 4,6 \\
\hline Coluna/medula & 1.145 & 2,7 & 742 & 2,7 & 403 & 2,7 \\
\hline Boca/dentes & 1.013 & 2,4 & 708 & 2,6 & 305 & 2,1 \\
\hline Pescoço & 988 & 2,4 & 629 & 2,3 & 359 & 2,4 \\
\hline Outros & 1.332 & 3,2 & 873 & 3,2 & 459 & 3,1 \\
\hline \multicolumn{7}{|l|}{ Evolução } \\
\hline Alta & 26.859 & 64,4 & 16.923 & 62,7 & 9.936 & 67,6 \\
\hline Internação & 7.444 & 17,9 & 5.204 & 19,3 & 2.240 & 15,3 \\
\hline Ambulatório & 5.658 & 13,6 & 3.705 & 13,7 & 1.953 & 13,3 \\
\hline Evasão & 275 & 0,7 & 201 & 0,7 & 74 & 0,5 \\
\hline Óbito & 90 & 0,2 & 75 & 0,3 & 15 & 0,1 \\
\hline Não informado & 1.351 & 3,2 & 881 & 3,3 & 470 & 3,2 \\
\hline Total & 41.677 & 100,0 & 26.989 & 100,0 & 14.688 & 100,0 \\
\hline
\end{tabular}

*Os dados não correspondem ao total, pois cada caso poderia ter mais de uma categoria preenchida concomitantemente.

psicoativas parecem estar associados aos comportamentos deste grupo etário ${ }^{9}$. Todavia, crianças menores de dez anos representaram uma parcela importante entreas vítimas de acidentes nesteestudo, principalmente entre as mulheres. Considerando o desenvolvimento infantil, as crianças apresentam dificuldade de localização exata de sons, sua visão periférica é restrita, tendem à distração e pre- cisam de mais tempo para processar informações. Tais características, somadas à inerente atividade de explorar 0 ambiente, contribuem para caracterizar as crianças como grupo vulnerável aos acidentes, 0 que requer permanentemente supervisão e orientação por parte dos adultos ${ }^{11,12}$.

Os negros (pretos e pardos) predominaram em todos os tipos de acidentes, confirmando as 
estatísticas encontradas no Brasil ${ }^{4,13,14}$. Segundo análises realizadas com dados do M inistério da Saúde ${ }^{13}$, a população negra apresenta os maiores coeficientes de mortalidade por causas externas. No período de2000 a 2003, estes coeficientes mantiveram-se estáveis entre os brancos, enquanto na população parda e preta observou-se crescimento no risco de morte por causas externas. Embora um grupo social não se defina por relações de raça ou cor, diferenças étnicas associam-se a desigualdades sociais e condicionam a forma de viver e de morrer degrupos populacionais. A etnia em si não é um fator de risco, mas a inserção social adversa de um grupo racial/étnico é que se constitui em característica de vulnerabilidade ${ }^{14}$. Outro aspecto importante foi a considerável proporção de atendimentos de pessoas com baixo nível de escolaridade, resultado semelhante ao apresentado em outros estudos nacionai ${ }^{15-17}$, nos quais também se verificou relação inversa entre a ocorrência de acidentes e o número de anos de estudo da vítima.

Em busca de atendimento, o meio de transporte mais utilizado para chegar ao serviço de emergência foi veículo particular, semel hanteao verificado no município do Rio de Janeiro, quando seanalisou a morbidade por acidentes de transporte em hospitais públicos ${ }^{18}$. No presente estudo, as ambulâncias aparecem em segundo lugar, incluindo as unidades de resgate do SAMU, principal componente da Política Nacional de Atenção às U rgências (PNAU) do Ministério da Saúde, cuja cobertura atinge 62 milhões de pessoas em 266 cidades brasileiras. As principais ações desta política incluem: a) organização do atendimento de urgência em unidades de pronto-atendimento; b) estruturação do atendimento préhospitalar móvel (SAM U/192); c) reorganização das grandes urgências e prontos- socorros em hospitais; d) criação de suporte hospitalar para os atendimentos de urgência; e) estruturação do atendimento pós-hospitalar ${ }^{19}$.

Com relação ao horário de ocorrência dos acidentes, no período da manhã, sua distribuição sofre influência das categorias outros tipos deacidentes e das queimaduras, enquanto os acidentes de transporte se sobressaem no período da tarde e noite. Em um estudo ${ }^{20}$ sobre a epidemiologia das hospitalizações por queimaduras, observou-se que elas foram mais frequentes entre as $10 \mathrm{~h}$ e $12 \mathrm{~h}$. Por outro lado, estudos realizados na região Sul do Brasil evidenciaram maior proporção de acidentes de transporteno período da tarde e início da noite ${ }^{21,22}$.

Dentre as causas externas, as quedas representam a principal causa de internações no sistema público de saúde brasileiro, principalmente entre crianças, adolescentes e idosos ${ }^{4}$. $\mathrm{N}$ a faixa etária de
0 a 9 anos, esse tipo deacidente poderia ser evitado se os pais ou responsáveis tivessem percebido os riscos antecipadamenteetomado providências para evitá-lo ${ }^{11,23}$. Entre os idosos, as quedas possuem um significado muito relevante, pois podem leválos à incapacidade, sequelas ou lesões permanentes e morte. Seu custo social é imenso e torna-se maior quando o idoso tem diminuição da autonomia e da independência ou passa a necessitar de institucionalização. N este grupo, deve-se val orizar o autocuidado, adaptações ambientais conforme as necessidades físicas do idoso e supervisão por parte dos familiares e cuidadores ${ }^{24,25}$.

Os meios de transporte que mais geraram vítimas foram a motocicleta e a bicicleta, resultados semelhantes aos encontrados no sistema de vigilância de causas externas de Cali, na Colômbia ${ }^{26}$. Detodos os atendimentos por estetipo deacidente, o condutor foi a vítima mais comum, seguido por passageiro e pedestre. Deve-se ressaltar quea categoria pedestre constitui-se, sobretudo, de crianças e idosos, grupos que apresentam maior restrição à sua mobilidade ${ }^{27}$. Por outro lado, a distribuição apresentada nos serviços de emergência incluídas no presente estudo é semelhante ao observado no estudo realizado em serviço de atendimento pré hospitalar de Londrina (Paraná), onde os motociclistas foram o principal tipo de vítima, com proporção superior a $40 \%$ do total de atendimentos de emergência por acidentes de transporte?.

Tanto os motociclistas como os ciclistas devem ser considerados mais vulneráveis em relação aos condutores de outros tipos de veículos. Quando os condutores de bicicletas são crianças e adolescentes, deve-se intensificar a atenção, pois, como a maioria deles utiliza a bicicleta como lazer, a consciência do risco a que estão expostos pode estar subestimada, ${ }^{9}$. Torna-se necessário desenvolver estratégias específicas para estes grupos, por meio de ações intersentoriais que incluam as áreas de segurança pública, engenharia de trânsito, saúde e educação.

As queimaduras são consideradas um importante problema de saúde pública, pois, além dos problemas físicos que podem levar o paciente à morte, elas acarretam danos de ordem psicológica e social ${ }^{29}$. No Brasil, os dados estatísticos sobre as lesões por queimaduras são escassos. Em 2005, foram registradas 60.451 internações por queimaduras no SIH, incluindo exposição à corrente elé trica, fogo esubstâncias quentes, enquanto no SIM foram registrados 2.421 óbitos por essas causas4. A exemplo dos resultados apresentados em outros estudos ${ }^{20,30}$, observou-se maior proporção de queimaduras em pessoas do sexo masculino, prin- 
cipalmente as queimaduras decorrentes de exposição a fogo e corrente elétrica, ocorridas geralmente no local de trabalho. Todavia, as queimaduras por substância quente foram marcantes no sexo feminino, devido, provavelmente, às atividades domésticas ou ao trabalho no setor de serviços ${ }^{31}$. Estes dados são importantes para que se possa compreender a magnitude do problema e identificar as populações mais atingidas e as circunstâncias nas quais as queimaduras ocorrem, de forma que seja possível implementar programas de prevenção29.

Os demais tipos de acidentes (cortes, queda de objetos sobre a pessoa, envenenamento, afogamento, sufocação, acidentes com arma de fogo e os não especificados) foram duas vezes mais fre quentes entre os homens. Embora tenham ocorrido em todas as faixas etárias, as crianças formam um grande grupo de risco para esses acidentes. Estes resultados são semelhantes aos apresentados em estudo envolvendo pacientes menores de 15 anos atendidos em prontos-socorros de Londrina (Paraná) ${ }^{32}$. Embora cada tipo de acidente tenha apresentado menor proporção em relação aos acidentes de transporte, quedas e queimaduras, eles não podem ser menosprezados, haja vista a parcela de carga social imputada ao sistema de saúde, bem como o sofrimento causado às vítimas e familiares.

A pesar de aproximadamente um terço dos acidentester ocorrido em via pública (36,2\%), chama a atenção a proporção de acidentes ocorridos no domicílio (33,7\%). Por ser o ambiente de maior permanência da maioria das pessoas, em especial as crianças, o principal local deocorrência dos acidentes é o próprio domicílio, sendo este o local de ocorrência da maioria das quedas, queimaduras, choques elétricos, contato com instrumentos cortantes, sufocação e afogamento 27,33 .

Outro aspecto a ser destacado na epidemiologia dos acidentes, sobretudo os relacionados aos meios de transporte, é o uso de substâncias psicoativas. Em pesquisa realizada com estudantes universitário ${ }^{34}$, menos de $4 \%$ reconheceram o hábito deingerir bebidas al coólicas como causador deseu envolvimento em acidentes de transporte; porém, entre os condutores que dirigiam após beber, 0 risco de envolvimento em acidentes de transporte foi $184 \%$ maior do que entre os que não apresentavam esse comportamento. Assim, o uso de álcool e outras drogas, associado à inexperiência, busca de emoções, prazer em experimentar sensações de risco e impulsividade são fatores associados ao comportamento de adolescentes e adultos jovens que podem contribuir para a maior incidência de acidentes, principalmente os de transporte 9 .

Neste estudo, os membros superiores foram a região do corpo mais afetada, enquanto na análise realizada no município de Londrina (Paraná) ${ }^{32}$, as lesões na região da cabeça e pescoço foram as mais frequentes. 0 destino do paciente após 0 atendimento de emergência pode ser empregado como medida indireta da gravidade da lesão ${ }^{27}$. Tanto a pequena proporção de óbitos como a relativa baixa ocorrência de internações hospitalares tornam evidente a má distribuição dos pacientes entre os serviços públicos deemergência e os de atenção primária, acarretando a superlotação dos primeiros.

Os dados aqui apresentados traduzem a realidade do cotidiano dos serviços de emergência e despertam novos olhares sobre o problema do atendimento às vítimas deacidentes. A maior contribuição da modalidade de vigilância de causas externas em serviços sentinelas é a disponibilização de dados em tempo hábil, de forma que sua análise einterpretação proporcionem as bases para a tomada de decisão. Outrossim, permite captar dados sobre eventos menos graves, mas cujo conhecimento é fundamental para o planejamento de políticas públicas de prevenção de agravos e promoção da saúde. 


\section{Colaboradores}

M DM M ascarenhas participou da concepção teórica, levantamento bibliográfico, consolidação e análise dos dados, elaboração e redação e revisão final do texto; M M A Silva, DM Carvalho, VP Gawryszewski e L M oura participaram da concepção teórica, análise dos dados, redação e revisão do texto até a versão final; VC Costa, M FM Souza e OL M orais N eto participaram da concepção teórica e revisão final do texto.

\section{Agradecimentos}

Agradecemos aos técnicos das secretarias estaduais e municipais de saúde, pela dedicação durante o treinamento e coleta de dados; aos pacientes atendidos nos serviços deemergência incluídos na Rede VIVA, pelo fornecimento das informações aqui apresentadas, mesmo em situação de sofrimento; e a Eduardo M arques Macário, pelo cuidadoso trabalho de identificação dos registros duplicados.

\section{Referências}

1. World Health Organization. Injury surveillance guidelines. Geneva: WHO; 2001.

2. World Health Organization. Guidelines for conducting community surveys on injuries and violence. Geneva: WH O; 2004.

3. World Health Organization. World report on violence and health. Geneva: WHO; 2002.

4. Brasil. M inistério da Saúde. Informações de saúde [site da Internet]. [acessado 2008 jan 10]. Disponível em: http://www.datasus.gov.br

5. Gawryszewski VP, Silva M MA, Malta DC, Mascarenhas MDM, Costa VC, M atos SG, M oraes Neto OL, M onteiro RA, Carvalho CG, Magalhães ML. A proposta da rede de serviços sentinela como estratégia de vigilância de violências e acidentes. Cien Saude Colet 2006; 11(Supl):1269-1278.

6. Brasil. M inistério da Saúde. Portaria n 737, de 16 de maio de 2001. Institui a política nacional de redução da morbimortalidade por acidentes e violências. Diário Oficial da União 2001; 18 mai.

7. Centers for Disease Control and Prevention. Epi Info, Version 3.3.2. Database and statistics software for public health professionals. Atlanta: CDC; 2004.

8. Malta DC, Cezário AC, M oura L, M orais N eto OL, Silva Jr JB. A construção da vigilância e prevenção das doenças crônicas não transmissíveis no contexto do Sistema Ú nico de Saúde. Epidemiol Serv Saúde 2006; 15(1):47-65.

9. Bastos YGL, Andrade SM, Soares DA. Características dos acidentes de trânsito e das vítimas atendidas em serviço pré-hospitalar em cidade do Sul do Brasil, 1997/2000. Cad Saude Publica 2005; 21(3):815-822.

10. Souza ER, M inayo MCS, Cavalcante FG. The impact of suicide on morbidity and mortality in the population of Itabira. Cien Saude Colet 2006; 11(2):409-418.

11. Deslandes SF, Assis SG, Santos NC. Violências envolvendo crianças no Brasil: um plural estruturado e estruturante. In: M inistério da Saúde. Impacto da violência na saúde dos brasileiros. Brasília: Ministério da Saúde; 2005. p. 43-78.

12. Goren S, Gurkan F, Tirasci Y, Kaya Z, Acar K. Foreign body asphyxiation in children. Indian Pediatrics 2005; 42(11):1131-1133.

13. Brasil. M inistério da Saúde. Saúde Brasil: uma análise da situação de saúde. Brasília: M inistério da Saúde; 2005.

14. Batista LE, Escuder M M L, Pereira JCR. A cor da morte: causas de óbito segundo características de raça no Estado de São Paulo, 1999 a 2001. Rev. Saude Publica 2004; 38(5):630-636.

15. M inayo MCS, Souza ER. Violência para todos. Cad Saude Publica 1993; 9(1):65-78.

16. Soares Filho AM, Souza M FM, Gazal-Carvalho C, Malta DC, Alencar AP, Silva M MA, Morais Neto OL. Análise da mortalidade por homicídios no Brasil. Epidemiol Serv Saúde 2007; 16(1):7-18.

17. Souza M FM, M alta DC, Conceição GMS, Silva M MA, Gazal-Carvalho C, M orais N eto OL. Análise descritiva e de tendência de acidentes de transporte terrestre para políticas sociais no Brasil. Epidemiol Serv Saude 2007; 16(1):33-44. 
18. Deslandes SF, Silva CM FP. Análise da morbidade hospitalar por acidentes de trânsito em hospitais públicos do Rio de Janeiro, RJ, Brasil. Rev. Saude Publica 2000; 34(4):367-372.

19. Malta DC, Lemos MSA, Silva MMA, Rodrigues MES, Gazal-Carvalho C, Morais N eto OL. Iniciativas de vigilância e prevenção de acidentes e violências no contexto do Sistema Único de Saúde (SUS). Epidemiol Serv Saúde 2007; 16(1):45-55.

20. Chien WC, Pai L, Lin CC, Chen CC. Epidemiology of hospitalized burns patients in Taiwan. Burns 2003; 29(6):582-588.

21. Scalassara MB, Souza RKT, Soares DFPP. Características da mortalidade por acidentes de trânsito em localidade da região Sul do Brasil. Rev. Saude Publica 1998; 32(2):125-132.

22. Barros AJD, Amaral RL, Oliveira MSB, Lima SC, Gonçalves EV. Acidentes de trânsito com vítimas: subregistro, caracterização e letalidade. Cad Saude Publica 2003; 19(4):979-986.

23. Gaspar VLV, Lamounier JA, Cunha FM, Gaspar JC. Fatores relacionados a hospitalizações por injúrias em crianças e adolescentes. J Pediatr (Rio J) 2004; 80(6) :447452.

24. Fabrício SCC, Rodrigues RAP, Costa Jr M L. Causas e consequências de quedas de idosos atendidos em hospital público. Rev. Saude Publica 2004; 38(1):93-99.

25. Minayo MCS. Violência contra idosos no Brasil: relevância para um velho problema. Cad Saude Publica 2003; 19(3):783-791.

26. Castro MB, Rendón LF, Rojas MC, Durán CA, Albornoz M. Caracterización de los pacientes con lesiones de causa externa mediante un sistema de vigilancia epidemiológica. Rev Colomb Cir 2006; 21(3):180-189.

27. Andrade SM, M ello Jorge MHP. Acidentes de transporte terrestre em município da Região Sul do Brasil. Rev. Saude Publica 2001; 35(3):318-320.

28. Soares DFPP, Barros M BA. Fatores associados ao risco de internação por acidentes de trânsito no M unicípio de M aringá-PR. Rev Bras Epidemiol 2006; 9(2):193-205.

29. Rossi LA, Barruffini RCP, Garcia TR, Chianca TCM. Queimaduras: características dos casos tratados em um hospital escola em Ribeirão Preto (SP), Brasil. Rev Panam Salud Pública/Pan Am. J. Public Health 1998; 4(6):401-404.

30. Fordyce TA, Kelsh M, Lu ET, Sahl JD, Yager JW. Thermal burn and electrical injuries among electric utility workers, 1995-2004. Burns 2007; 33(2):209-220.

31. Islam SS, Nambiar AM, Doyle EJ, Velilla AM, Biswas RS, Ducatman AM . Epidemiology of work-related burn injuries: experience of a state-managed workers' compensation system. J Trauma 2000; 49(6):1045-1051.

32. M artins CBG, Andrade SM. Causas externas entre menores de 15 anos em cidade do Sul do Brasil: atendimentos em pronto-socorro, internações e óbitos. Rev Bras Epidemiol 2005; 8(2):194-204.

33. Paes CEN, Gaspar VLV. As injúrias não intencionais no ambiente domiciliar: a casa segura. J Pediatr (Rio J) 2005; 81(5 Supl):S146-S154.

34. M arín-León L, Vizzotto M M. Comportamentos no trânsito: um estudo epidemiológico com estudantes universitários. Cad Saude Publica 2003; 19(2):515-523.

Artigo apresentado em 23/04/2007

Aprovado em 15/02/2008

Versão final apresentada em 04/03/2008 